\title{
Medium-Range Structure and Glass Forming Ability in Zr-Cu-Al Bulk Metallic Glasses
}

\author{
Pei Zhang ${ }^{1}$, Jason J. Maldonis ${ }^{1}$, M.F. Besser ${ }^{2}$, M.J. Kramer ${ }^{2}$, Paul M. Voyles ${ }^{1 *}$
}

${ }^{1}$ Department of Materials Science and Engineering, University of Wisconsin, Madison, Madison, Wisconsin 53706, USA

${ }^{2}$ Ames Laboratory (DOE), Ames, Iowa 50011, USA and Department of Materials Science and Engineering, Iowa State University, Ames, Iowa 50011, USA

\begin{abstract}
Fluctuation electron microscopy experiments combined with hybrid reverse Monte Carlo modeling show a correlation between medium-range structure at the nanometer scale and glass forming ability in two $\mathrm{Zr}-\mathrm{Cu}-\mathrm{Al}$ bulk metallic glass (BMG) alloys. Both $\mathrm{Zr}_{50} \mathrm{Cu}_{35} \mathrm{Al}_{15}$ and $\mathrm{Zr}_{50} \mathrm{Cu}_{45} \mathrm{Al}_{5}$ exhibit two nanoscale structure types, one icosahedral and the other more crystallike. In $\mathrm{Zr}_{50} \mathrm{Cu}_{35} \mathrm{Al}_{15}$, the poorer glass former, the crystal-like structure is more stable under annealing below the glass transition temperature, $T_{g}$, than in $\mathrm{Zr}_{50} \mathrm{Cu}_{45} \mathrm{Al}_{5}$. Variable resolution fluctuation microscopy of the MRO clusters show that in $\mathrm{Zr}_{50} \mathrm{Cu}_{35} \mathrm{Al}_{15}$ on sub- $T_{g}$ annealing, the crystal-like clusters shrink even as they grow more ordered, while icosahedral-like clusters grow. Overall, the results suggest that achieving better glass forming ability in this alloy system may depend more on destabilizing crystal-like structures than enhancing non-crystalline structures.

Key words: fluctuation electron microscopy, medium range order, metallic glass, glass forming ability, hybrid reverse Monte Carlo

Corresponding author: Paul M. Voyles Email address: paul.voyles@wisc.edu
\end{abstract}




\section{Introduction}

Bulk metallic glasses (BMGs) show a variety of outstanding properties including high yield strength [1], a high elastic limit in tension or compression [2,3], and excellent nanoprocessability [4]. However, high critical cooling rates limit the formation and applications of many BMGs, so enhancing the glass-forming ability (GFA) and understanding the origin of good GFA are important to further applications of these alloys. Understanding GFA may also advance basic scientific understanding of the glass transition and crystal nucleation and growth. Inoue [5] proposed three famous empirical rules for BMG formation and better GFA: a multicomponent system, atomic size ratios above $12 \%$, and negative heats of mixing between constituent elements. Other researchers have proposed various thermodynamic and kinetic parameters to describe GFA, including high reduced glass transition temperature, $T_{r g}=T_{g} / T_{m}$, where $T_{m}$ is the melting temperature; large width of supercooled liquid region $\Delta T_{x}=T_{x}-T_{g}$, where $T_{x}$ is the crystallization temperature [6]; and large $\gamma=T_{x} /\left(T_{g}+T_{l}\right)$ where $T_{l}$ is the liquidus temperature $[7,8]$. Still other researchers emphasize short-range structural order in the form of efficient packing [9-11], low atomic-level stress [12], or a high degree of icosahedral bond topology [13,14]. In general, these rules and parameters are consistent with most but not all known glass formers and have some success predicting the GFA of new alloys. However, none of them predict every known glass former, nor have any of them been widely accepted as a reliable tool for predicting new metallic glass compositions.

Here, we study the relationship of structure and structural stability with GFA. Much of our current understanding of these connections comes from simulations, especially molecular dynamics (MD). Previous simulation work has identified solute-centered nearest-neighbor clusters as important short range order (SRO) building blocks of BMG structure [15]. These clusters are categorized by the their Voronoi polyhedron (VP) indices, and the icosahedron is the dominant unit in BMGs such as $\mathrm{Zr}-\mathrm{Cu}$ [16], Ni-Nb [17], Ni-Zr [18], Zr-Pd [19], and Zr-Cu$\mathrm{Al}[20]$. From a geometric viewpoint, icosahedra with five-fold symmetry are preferred SRO units due to their efficient packing when the atomic size ratio between solute atoms and solvent atoms is close to 0.902 [21-24]. However, in 3D space their fivefold rotational symmetry is incompatible with long range translational symmetry [25]. Distorted icosahedra with partial fcc symmetry can pack in 3D [26]. MD simulations show that icosahedra with high packing density lead to low configurational potential energy and low entropy $[27,28]$.

Icosahedral order may also stabilize the supercooled liquid phase. In MD, the density of icosahedral SRO clusters increases rapidly with cooling inside the supercooled liquid, and the atoms involved in icosahedral clusters exhibit slower dynamics than other atoms [13,14]. The existence of icosahedral order may also increase the barrier of nucleation and lead to slower structural rearrangement [29,30]. Alloying of $\mathrm{Al}$ into $\mathrm{Zr}-\mathrm{Cu}$ BMGs increases the packing density, further stabilizing the icosahedral order and causing the corresponding crystal to be less competitive, which enhances GFA [20,31].

Medium range order (MRO), which occupies the length scale between SRO and longrange order, can be thought of as packing of SRO. In the efficient cluster packing model [10,32], MRO is determined by the efficient packing of solute-centered clusters by sharing solvent atoms. In MD simulations SRO clusters connect via face-, edge-, or vertex-sharing without strong ordering at the MRO length scale [10,15,20]. Recently, Hwang et al. [33] provided a new model for the atomic structure of $\mathrm{Z}_{50} \mathrm{Cu}_{45} \mathrm{Al}_{5} \mathrm{BMG}$ based on incorporating experimental data on 
MRO from fluctuation electron microscopy (FEM) with an empirical interatomic potential using hybrid reverse Monte Carlo (HRMC) modeling. The models show that the majority of VP in $\mathrm{Zr}_{50} \mathrm{Cu}_{45} \mathrm{Al}_{5}$ BMG can be classified into two groups: 1) icosahedral-like clusters with approximate five-fold rotational symmetry and 2) crystal-like clusters with approximate fourand six-fold rotational symmetry. Similar VP group together to form larger superclusters on the MRO length scale. The icosahedral-like clusters form nanoscale chains, while the crystal-like clusters form blobs which exhibit overall four- and six-fold symmetry. A transformation from crystal-like to icosahedral-like clusters is observed after structural relaxation by annealing below the glass transition temperature, $T_{g}[33]$.

In this paper, we combine FEM experiments and HRMC simulations to study the SRO and $\mathrm{MRO}$ of $\mathrm{Zr}_{50} \mathrm{Cu}_{35} \mathrm{Al}_{15} \mathrm{BMG}$, a poorer glass former than $\mathrm{Zr}_{50} \mathrm{Cu}_{45} \mathrm{Al}_{5}$ [34]. The new alloy exhibits the same two icosahedral-like and crystal-like structure types, but differs in its details and thermal stability. We describe correlations between the nanoscale structure and structural stability in the glassy state with GFA, and suggest that efforts to improve GFA should include consideration of destabilizing crystal-like structures as well as enhancing icosahedral structures.

\section{Experimental and computational methods}

\section{Samples and sample preparation of $\mathbf{Z r}_{50} \mathbf{C u}_{35} \mathbf{A l}_{15}$}

A mixture of the component elements of 99.9 at.\% purity or higher was arc-melted in a Ti-gettered, high-purity argon atmosphere to make a master ingot. The ingot was then remelted and injected onto a copper wheel spinning at $25 \mathrm{~m} / \mathrm{sec}$ tangential wheel speed, forming ribbons with $\sim 400 \mu \mathrm{m}$ thick and $0.5 \mathrm{~cm}$ wide. Samples were annealed at $0.83 T_{\mathrm{g}}$ for $10 \mathrm{~min}$ and $60 \mathrm{~min}$ to measure the MRO after structural relaxation.

$T_{g}$ and $T_{x}$ for the as-cast alloy were measured using a TA Instruments Q100 Modulated Differential Scanning Calorimeter (DSC) with an autosampler at a heating rate of $0.33 \mathrm{~K} / \mathrm{s}$ under a flow of purified argon. Annealing for structural relaxation was done in the DSC with an argon environment at $573 \mathrm{~K}\left(0.83 T_{\mathrm{g}}\right)$ for $10 \mathrm{~min}$ and $60 \mathrm{~min}$. For annealed samples, we performed a pre-annealing at $473 \mathrm{~K}$ for $1 \mathrm{~min}$ to ensure thermal stability.

$\mathrm{Zr}_{50} \mathrm{Cu}_{35} \mathrm{Al}_{15}$ TEM samples were prepared by electropolishing. The electrolyte was composed of $\mathrm{HNO}_{3}(9 \%)$ with butyl cellosolve + methanol mixture (1:2). The temperature range during the electropolishing was between $-42{ }^{\circ} \mathrm{C}$ and $-52{ }^{\circ} \mathrm{C}$. Samples were electro-polished at 42 $\mathrm{V}$ using a Struers Tenupol electropolisher [35,36] with a current between 60 and $75 \mathrm{~mA}$. Electropolishing forms a thick oxidation layer on the surface of the sample, so after electropolishing we employed low energy ion milling (Fischione 1040 Nanomill) to remove the oxidation layer. On both sides of the TEM sample, a $60 \mu \mathrm{m}$ x $25 \mu \mathrm{m}$ box was milled at $-168{ }^{\circ} \mathrm{C}$ with $700 \mathrm{eV}$ for 20 minutes. Immediately before FEM measurements, TEM samples were plasma cleaned at $20 \mathrm{psi} \mathrm{Ar}+\mathrm{O}_{2}$ mixture for $\sim 30 \mathrm{~s}$.

\section{FEM measurements}

FEM is an effective way to detect MRO structure through spatial fluctuations in coherent electron nanodiffraction, which are sensitive to three and four-body correlation functions [3740]. FEM studies the statistically normalized variance $V$ of the spatially-resolved diffracted intensity $I$ of many nanodiffraction patterns measured in a scanning transmission electron microscope (STEM) as a function of scattering vector magnitude $k$ and the coherent spatial resolution $R$. $V$ is defined as 


$$
V(k, Q)=\frac{\left\langle I^{2}(k, Q, r)\right\rangle}{\langle I(k, Q, r)\rangle^{2}}-1,
$$

where $<>$ indicates averaging over the position on the sample. The spatial resolution $R$ (typically 1-2 $\mathrm{nm}$ ) can be defined by the Raleigh criterion, $R=0.61 / Q$ [38], in which $Q$ is the radius of the virtual objective aperture in reciprocal space.

FEM experiments were performed in an FEI Titan STEM with a probe $\mathrm{C}_{\mathrm{s}}$ aberration corrector at $200 \mathrm{kV}$. The Schottky emission gun was operated at $4500 \mathrm{~V}$ extraction voltage. Microprobe mode ( $\mu \mathrm{P}$-mode) of the FEI control software with the extended $Q$ range was chosen and a camera length of $512 \mathrm{~mm}$ in energy filtered STEM (EF-STEM) mode with a $10 \mathrm{eV}$ wide slit was used. A Gatan annular dark-field detector mounted on the $2.5 \mathrm{~mm}$ GIF entrance aperture was used to collect STEM images simultaneously with the nanodiffraction patterns. The diffraction lens current was adjusted using the FEI free-lens control interface to ensure good diffraction focus. $512 \times 512$ pixel nanodiffraction patterns were acquired with a CCD binning of four on a $10 \times 10$ grid of positions covering a $30 \times 30 \mathrm{~nm}^{2}$ area of the sample. Variance data from ten different areas of each sample were averaged together and reported with one standard deviation of the mean error bars. The exposure time was set to $6 \mathrm{~s}$ in order to ensure a sufficiently high signal to noise ratio. The sample thickness was measured using the log-ratio method applied to the elastic scattering transmittance. We used the same elastic mean free path as was used in the $\mathrm{Zr}_{50} \mathrm{Cu}_{45} \mathrm{Al}_{5} \mathrm{BMG}, 39 \pm 1 \mathrm{~nm}$ [41]. Typically, the samples had an electron transmittance of $40 \% \sim 50 \%$, which is thin enough for FEM experiments [36].

Despite the precautions described above, a surface oxidation layer covers the sample even after low energy ion milling. Figure 1a shows $V(k)$ for the as-cast BMG sample acquired from both the thin and thick regions. The thinnest region exhibits only one peak at $0.33 \AA^{-1}$, lower in $k$, which corresponds reasonably well to the lowest order reflections from $\mathrm{Zr}$ and $\mathrm{Cu}$ oxides, so we assign them to the surface oxide. The thicker region exhibits peaks at $0.33 \AA^{-1}$ arising from metal-oxygen-metal bonding and peaks at $0.41 \AA^{-1}$ and $0.45 \AA^{-1}$ arising from metalmetal bonds, and thus the BMG. The elastic transmittance shows that the oxide-only region is $\sim 20 \mathrm{~nm}$ thick and the thicker region is $\sim 50 \mathrm{~nm}$ thick, so there is $\sim 30 \mathrm{~nm}$ of residual metal. If plural elastic scattering is small, to a good approximation, $V(k)$ from the oxide and the metal simply add. We therefore obtain the signal from just the metal by measuring the signal from just the oxide, then subtracting it from the combined metal/oxide signal, as shown in Figure 1b. Similar measurements of thin, fully oxidized regions, and thicker, partially oxidized regions, are applied to the $V(k)$ data for samples annealed for $10 \mathrm{~min}$ and $60 \mathrm{~min}$.

By adjustment of the three full strength condenser lenses, the probe convergence angle $Q$, and thus the probe size $R$, can be varied with fixed condenser aperture, enabling variable resolution FEM (VRFEM). VRFEM is the study of $V(Q)$ at fixed $k$. That data can be analyzed in the context of the pair persistence model [42] to yield the decay length $\Lambda$ of an approximate four-body atom position correlation function. $\Lambda$ is then a measure of the size of the MRO causing diffraction at a particular $k$. The pair persistence model predicts that $1 / V$ vs $1 / Q^{2}$ is a straight line, and $\Lambda$ is derived from the line slope and intercept [42]. We varied the probe size from $1.3 \mathrm{~nm}$ to $11.0 \mathrm{~nm}$ with nearly constant coherence [39]. The parameters for each probe size are given in Table 1. $V(Q)$ data are not corrected for the surface oxide.

\section{Computational methods HRMC}


Structures for $\mathrm{Zr}_{50} \mathrm{Cu}_{35} \mathrm{Al}_{15}$ in various states of relaxation were derived from HRMC simulations [33,43-46]. The HRMC simulations were constrained by SRO from an empirical interatomic potential and MRO from FEM data. The HRMC algorithm uses Metropolis Monte Carlo with random single-atom moves to minimize a cost function $C=\alpha \chi^{2}+E$, were $E$ is the system potential energy, and $\chi^{2}$ measures the discrepancy between the simulated variance $V_{s}(k)$ and the experimental variance $V(k) . \chi^{2}$ is defined by

$$
\chi^{2}=\sum_{i}\left[\frac{V\left(k_{i}\right)-\beta \cdot V_{s}\left(k_{i}\right)}{\sigma_{e r r}}\right]^{2}
$$

in which $\sigma_{e r r}$ is the experimental error for each $V(k)$ data point and $\alpha$ is a weighting factor set to encourage energy minimization while constraining the fit of the experimental data within reasonable values (based on the experimental error). $\beta=\frac{1}{3} \frac{t_{\text {sim }}}{t_{\text {exp }}}$ is a scaling factor that corrects for the thickness difference between the sample and the model and for the approximations in the $V(k)$ calculation $[33,47]$. The interatomic potential for $\mathrm{Zr}-\mathrm{Cu}-\mathrm{Al}$ was developed by $\mathrm{Cheng}$ et al. [20], then modified later by H. Sheng [48]. Hwang et al. used the original version of the potential.

MD in the NPT ensemble at zero pressure in LAMMPS was used to determine the equilibrium density of the $\mathrm{Zr}_{50} \mathrm{Cu}_{35} \mathrm{Al}_{15}$ glass state at room temperature predicted by the potential. A $2.83^{3} \mathrm{~nm}^{3}$ box was then randomly filled with atoms with the same atom density and the correct composition, and conjugate gradient minimization in LAMMPS was used to find a local minimum of the energy of the structure. The resulting dense random packed structure was used as the input model for each HRMC simulation for the three sample states.

The HRMC simulations calculate the intensity of the FEM measurement $I(k)$ using the Dash et al. method [47]. To obtain enough samples from which to calculate $V, I$ is calculated with the incident electron beam traveling along 211 different directions, evenly spaced in solid angle on the top half of the unit circle. This approach is equivalent to duplicating the model many times with random rotations to make a larger structure, and it helps enforce orientational isotropy on the final structure. In the simulation, the pixels are modified from circular in shape to square, and the model size $s$ is set by the experiment resolution $R$ by $s=\sqrt{2} R(R=2 \mathrm{~nm}$ in this case). The combination of square pixels and the model size ensures each atom in the model contributes once and only once to the $I(k)$ calculation in each orientation.

The simulation minimizes the cost function using random, single-atom moves accepted or rejected according to the Metropolis algorithm and an effective temperature. The temperature is decreased in a simulated annealing framework after the cost function has equilibrated at a given temperature. Figure 2 shows the convergence of the cost function, energy, $\chi^{2}$, and the decrease of temperature as a function of step number for the $300{ }^{\circ} \mathrm{C}, 60 \mathrm{~min}$ annealed sample. The result is a model that is close in energy to the MD quenched glass and also in excellent agreement with the experimental FEM data. Minimizing the cost function requires $\sim 10^{7}$ random moves, which, using 64 cores, takes approximately five days to complete on modern computer architecture.

HRMC simulations were performed against the oxide-subtracted $V(k)$ data sets with $R=2$ $\mathrm{nm}$ only. The experimental sample thickness, $t_{\text {exp }}$, was $27.0 \mathrm{~nm}$ and $t_{\text {sim }}$ was $2.83 \mathrm{~nm}$, giving $\beta=0.0349$. The weighting parameter between $V(k)$ and $V_{s}(k)$ was $\alpha=40$ for consistency with [33].

\section{Model structure analysis}


We developed a reciprocal space method to visualize and analyze the structure of the HRMC models. Because FEM is a diffraction technique, the structure it imposes on the HRMC models is well-visualized in reciprocal space. An FCC aluminum crystal inside a larger box illustrates this process in Figure 3. First, the model is transformed into reciprocal space (Figure $3 b)$ via a three-dimensional Fourier transform (FT),

$$
S(\boldsymbol{k})=\sum_{n} f_{n}(|\boldsymbol{k}|) e^{2 \pi i \boldsymbol{k} \cdot \boldsymbol{r}_{n}},
$$

where $f_{n}$ is the atomic scattering factor for atom $n, \boldsymbol{k}$ is the wavevector, and $\boldsymbol{r}_{\boldsymbol{n}}$ is atom $n$ 's position. The complex vector $S(\boldsymbol{k})$ is not accessible experimentally in conventional experiments which only measure the real part of the function after isotropic averaging to yield $|S(k)|$. High intensity spots in the 3D FT are found using conventional feature analysis techniques implemented in Igor Pro software, and fitting to an asymmetric 3D Gaussian refines their positions and size. The functional form of an asymmetric 3D Gaussian is given by

$$
\begin{aligned}
G(x, y, z)= & \exp \left[\frac{1}{2}\left(1-c_{x y}{ }^{2}-c_{x z}{ }^{2}-{c_{y z}}^{2}+2 c_{x y} c_{x z} c_{y z}\right)^{-1}\right. \\
& \left.* \sum_{\substack{i, j, k=\\
x, y, z}}\left[\left(c_{i j}{ }^{2}-1\right) \frac{\left(k-k_{0}\right)^{2}}{\sigma_{k}{ }^{2}}+\frac{2\left(i-i_{0}\right)\left(j-j_{0}\right)\left(c_{i j}-c_{i k} c_{j k}\right)}{\sigma_{i} * \sigma_{j}}\right]\right]
\end{aligned}
$$

where the summation over $i, j, k=x, y, z$ indicates cycling through the index order.

To connect reciprocal space back to real space, we use Fourier filtering. A Gaussian windowing function is applied to the spot $\mathbf{g}$ and its symmetric reflection -g, filtering out all other diffraction phenomena (Figure 3b). The filtered 3D image is back-transformed into real space (Figure 3c) via

$$
|F(\boldsymbol{r})|=\sum_{\boldsymbol{k}}\left|S(\boldsymbol{k}) e^{-2 \pi i \boldsymbol{k} \cdot \boldsymbol{r}}\right| .
$$

$|F(\boldsymbol{r})|$ is a continuous function describing the electron scattering power of the sample, analogous to the electron density map obtained in x-ray crystallography. The result of the windowed back transform is a set of fringes perpendicular to $\mathbf{g}$, with periodicity $2 /|\mathbf{g}|$. The amplitude of the fringes is highest in the section of the model that contains atoms separated by $2 /|\mathbf{g}|$, so smoothing the back transforms highlights that section of the model (Figure 3d). This method is applied to the 3D structures of the $\mathrm{Zr}_{50} \mathrm{Cu}_{35} \mathrm{Al}_{15}$ models to identify MRO structures that give rise to particular features in $V(k)$ for further analysis. This method enables us to connect specific features in $V(k)$ with structures in the model.

Models were also analyzed using radial distribution functions calculated using R.I.N.G.S. [49] and VP indices [50]. VP indices were grouped into three categories: crystal-like, icosahedra-like, and mixed as defined in Table 2 following Hwang et al. [33].

\section{Experimental results}

Figure 4 shows heat release from DSC data for the as quenched and annealed samples. The as-quenched sample shows substantial heat release due to structural relaxation below $T_{g}$. The two annealed samples show substantially less heat release and are thus in a lower enthalpy state before the DSC measurement.

Figure 5 shows $V(k)$ after oxide subtraction for the as-cast and annealed samples for $R=2$ nm. One main peak exists at $k \sim 0.41 \AA^{-1}$, with shoulders near $k \sim 0.37 \AA^{-1}$ and $k \sim 0.45 \AA^{-1}$. After structural relaxation, the amplitude of the peak at $\sim 0.37 \AA^{-1}$ increases substantially. The peak at $k \sim 0.41 \AA^{-1}$ increases slightly after annealing for $10 \mathrm{~min}$, then decreases after annealing 

for 60 min but the changes are not consistently outside the experimental uncertainty. The peak at $k \sim 0.45 \AA^{-1}$ remains nearly constant.

Figure 6a shows the change in $V(k)$ (without oxide subtraction) with probe size for the ascast alloy. The magnitude of $V$ for all three peaks gradually decreases as the probe size increases. When the probe diameter becomes relatively large $(R=8 \mathrm{~nm}$ and $11 \mathrm{~nm}), V$ becomes small and the variation with $k$ becomes very small, indicating that nanodiffraction and thus the sample structure are nearly homogeneous at this length scale. Figure $6 \mathrm{~b}$ shows the pair persistent model fitting results of $\Lambda$ for the three peaks identified in Figure 5. $R=11 \mathrm{~nm}$ is removed from the fitting for peaks at $k \sim 0.37 \AA^{-1}$ and $k \sim 0.45 \AA^{-1}$ because variance from real structural information at this resolution is very small compared to variance induced by background sources like small thickness variations or other TEM sample imperfections and the oxide signal. The Pearson correlation $R^{2}$ for $k \sim 0.37 \AA^{-1}$ fitting changes from 0.989 to 0.897 if resolution of $R=11$ $\mathrm{nm}$ is included.

Similar VRFEM experiments and fitting of $\Lambda$ for all three peaks were carried out for samples annealed for $10 \mathrm{~min}$ and $60 \mathrm{~min}$, shown in Figure 7 and Figure 8 respectively. Compared with the VRFEM results of the as-cast sample at different resolutions, the magnitude of the peak at $k \sim 0.37 \AA^{-1}$ becomes larger and the peaks at $k \sim 0.41 \AA^{-1}$ and $k \sim 0.45 \AA^{-1}$ remain nearly unchanged. The fitting results of $\Lambda$ for different samples are summarized in Table 3 . After structural relaxation, the decay length increases for the peak at $k \sim 0.37 \AA^{-1}$, decreases for the peak at $k \sim 0.41 \AA^{-1}$, and remains unchanged for peak at $k \sim 0.45 \AA^{-1}$. The evolution of $\Lambda$ indicates that structural relaxation mainly occurs during heating and/or the early stage of annealing (first $10 \mathrm{~min}$ ). All the fitting results are shown in Table 3.

\section{Simulation results}

Figures 5a-c show the final simulated variance $V_{s}(k)$ for the three samples at the end of the HRMC structure refinement, with residuals plotted above. All three simulated data are in excellent agreement with the corresponding $V(k)$, with a maximum residual of $4.7 \%$. Table 4 shows the average atomic energies of the final HRMC models compared to the energy of the MD quenched model. The HRMC model energies are consistently larger than the MD model energies due to the competition of minimizing the energy vs. minimizing $\chi^{2}$, but they are all within a few $k T$ at room temperature.

Figure $5 \mathrm{~d}$ shows the total simulated $\mathrm{x}$-ray radial distribution functions for the three final models. All three are essentially identical, indicating similar SRO structures. Figure 9 shows an atomic rendering of a region of the final HRMC model for the $60 \mathrm{~min}, 300{ }^{\circ} \mathrm{C}$ annealed sample. The orientation of the model was selected to emphasize the ordered, high rotational symmetry section in the center of the model. Atomic coordinates for all three models are available as supplementary information.

Ten additional HRMC runs for the $60 \mathrm{~min}, 300{ }^{\circ} \mathrm{C}$ annealed sample were conducted using different random number generator seeds but with the same starting structure to illustrate the robustness of the HRMC approach. The resulting structures are not identical, but they are qualitatively and statistically similar. All ten have the same disordered and planar regions, and, as shown in Figure 10, the VP results for the ten models are statistically similar within the expected $\sqrt{n}$ variability expected for counting (Poisson) statistics. These results indicate that while there are many unique atom configurations that agree equally well with the FEM experimental data, the key features of mixed crystal-like / icosahedral-like regions are common to all of them. 


\section{Discussion}

Figure 11a shows that the fraction of crystal-like VP increases with annealing while the number of icosahedral-like VP decreases. This indicates that the planar structure in $\mathrm{Zr}_{50} \mathrm{Cu}_{35} \mathrm{Al}_{15}$ becomes more crystal-like with annealing at the cost of icosahedral-like local structures. This is also in contrast to the $\mathrm{Zr}_{50} \mathrm{Cu}_{45} \mathrm{Al}_{5}$ glass in which the number of icosahedral-like VP increases with annealing and the number of crystal-like VP decreases, suggesting that the crystal-like clusters are more stable and the icosahedral-like clusters are less stable in the poorer glass former than the better glass former. In addition, while the fractions of VP types in both compositions are broadly similar, the $\mathrm{Zr}_{50} \mathrm{Cu}_{35} \mathrm{Al}_{15}$ models have more crystal-like clusters overall than $\mathrm{Zr}_{50} \mathrm{Cu}_{45} \mathrm{Al}_{5}$, as well as a mean coordination number with one less one atom (11.8 vs. 12.8). The change in mean coordination number implies an overall shift in the local topology of the glasses, which is consistent with the change in composition. In MD simulations of similar MGs [20,51], the fraction of icosahedral-like VP indexes is approximately twice that found for HRMC generated models.

Despite the increasing number of crystal-like VP with annealing, the size of the crystallike region in the HRMC models does not change significantly. This indicates that the crystallike region instead becomes more ordered during annealing. Due to the relatively small size of our simulation models, it is likely not possible for the crystal-like region to grow to larger sizes while correctly reproducing the experimental data and retaining a reasonable energy.

At first glance, the VRFEM results in Table 3 seem to tell a different story. The results show that the crystal-like regions shrink while the icosahedral-like regions grow with annealing. This apparent conflict with the simulation results is resolved in two ways. First, it is likely that our HRMC models are too small to accurately model the size of the crystal-like clusters; each model contains precisely one crystal-like cluster near the center of the supercell, so we suspect that the size is constrained by the periodic boundary conditions. Unfortunately, the FEM calculation requires the model size to be an integer multiple of the probe size, and refining a $4 \times 4 \times 4 \mathrm{~nm}$ model is beyond our current computational capabilities.

Second, and more interesting, VRFEM and the VP statistics measure different aspects of the structure. VRFEM is primarily sensitive to the size of ordered regions, whereas the VP statistics are also sensitive to the degree or perfection of the structural order. Annealing could therefore make the crystal-like cluster smaller by converting atoms near the surface of the planar structure to icosahedral-like, mixed, or uncategorized and simultaneously make it more crystallike by converting atoms on the interior from mixed or icosahedral-like to crystal-like. The VRFEM size decreases at the same time the fraction of crystal-like VP increases. This interpretation is consistent with the increase in crystal-like VP and decrease in mixed VP in Figure 11 and the lack of change in the $\Lambda$ associated with the mixed-character peak at $0.45 \AA^{-1}$.

Figure 12 illustrates the 3D FT analysis for the $60 \mathrm{~min}, 300{ }^{\circ} \mathrm{C}$ annealed sample. Figure $12 \mathrm{a}$ and $\mathrm{b}$ are isosurface images of the 3D FT, which identify volumes in reciprocal space where significant diffraction occurs. Figure 12c shows the most important atoms that contribute to the diffraction of the spot-pair circled in Figures 12a and $b$, identified using the filtering, back transform, and smoothing method discussed above. This "sub-model" consists primarily of an ordered cluster of approximately 300 atoms near the center of the supercell, previously identified by hand in Figure 9, along with some surrounding atoms. Each of the three pairs of the most intense diffraction spots in Figures $12 \mathrm{a}$ and $\mathrm{b}$ result in a sub-model consisting of mostly the same 
atoms. This indicates that each spot-pair results from a different orientation of the same crystallike structure, which has approximate 6-fold rotational symmetry.

The vector $\mathbf{g}$ from the center of the 3D FT to each spot is perpendicular to the real space planes that cause the diffraction. By rotating the sub-model in Figure 12c according to the orientation of $\mathbf{g}$, we can identify exactly which set of planes gives rise to a specific pair of spots. This planar structure is shown (with planes vertical) in Figure 12d. We can connect spots to peaks in $V(k)$ by comparing the length of various g's to peak positions in $k$. Figure 13 shows the normalized intensity as a function of length $|\mathbf{g}|$ for all the spots identified in the computed reciprocal space superimposed on $V(k)$ for the as-quenched sample. The vertical axis is the integrated intensity inside each 3D spot, divided by the average intensity of a shell with the same radius $|\mathbf{g}|$. (The 3D FT is not calculated with experimental convergence, collection angles, or electron scattering factors, so the raw spot intensities are not physically meaningful.) The high intensity spots are clustered around the main peak in $V(k)$ and its shoulders, and there is one strong peak associated with the high $k$ peak centered at $0.7 \AA^{-1}$. The strong correspondence between the spot positions and $V(k)$ peaks emphasizes the origin of the planar structures in the model as arising from the HRMC algorithm forcing the model to match the FEM data.

The character of the VP in the sub-models for each $|\mathbf{g}|$, connected by Figure 12 to features in $V(k)$, enable us to make assignments of peaks in $V(k)$ to generalized structural features in the models. As diagnostics, we use $f_{i c o}, f_{x t a l}$, and $f=f_{\text {ico }} / f_{x t a l} . f_{i c o}$ is the ratio of the number of icosahedral-like atoms in a particular sub-model to the number of icosahedral-like atoms in the full model, so it represents the fraction of the icosahedral-like atoms in the full model captured by the sub-model. $f_{x t a l}$ is analogous for crystal-like atoms, and $f$ gives a measure of the relative icosahedral-like or crystal-like character of the sub-model. Figure 14 shows $f$ as a function of $|\mathbf{g}|$ for all three sample states. The data are quite scattered, but there is a trend from more icosahedral- to more crystal-like with increasing $k$. The trend grows more pronounced with annealing, and is strongest for the low and high $k$ shoulders on the main peak in $V(k)$. We therefore identify the right-hand should near $0.45 \AA^{-1}$ as arising from the more crystal-like structure, and the left-hand should near $0.37 \AA^{-1}$ as more icosahedral-like. The main peak near $0.41 \AA^{-1}$ is less clearly defined, but it becomes more crystal-like with annealing and so we identify it as primarily crystal-like. The assignments of the low $k$ shoulder and main peak are the same as what Hwang et al. found by hand for $\mathrm{Zr}_{50} \mathrm{Cu}_{45} \mathrm{Al}_{5}$ [33], but now backed by quantitative analysis. Hwang et al. did not consider the high- $k$ shoulder.

Figure 15 compares $V(k)$ for the $\mathrm{Zr}_{50} \mathrm{Cu}_{35} \mathrm{Al}_{15}$ and $\mathrm{Zr}_{50} \mathrm{Cu}_{45} \mathrm{Al}_{5}$ BMGs [33] after $300{ }^{\circ} \mathrm{C}$, $60 \mathrm{~min}$ annealing. Although the peak positions are not in exactly the same position, likely due to different mean interatomic distances arising from the difference in composition, $\mathrm{Zr}_{50} \mathrm{Cu}_{45} \mathrm{Al}_{5}$ shows the same low- $k$ shoulder, main peak, high- $k$ shoulder pattern as $\mathrm{Zr}_{50} \mathrm{Cu}_{35} \mathrm{Al}_{15}$. The high- $k$ shoulder in particular is much lower in $\mathrm{Zr}_{50} \mathrm{Cu}_{45} \mathrm{Al}_{5}$ than $\mathrm{Zr}_{50} \mathrm{Cu}_{35} \mathrm{Al}_{15}$ and the low- $k$ shoulder is much higher. The main peak is similar in magnitude. Given the structure assignments for the shoulders above, Figure 15 shows that after structural relaxation, $\mathrm{Zr}_{50} \mathrm{Cu}_{45} \mathrm{Al}_{5}$ has stronger icosahedral-like character and weaker crystal-like character than $\mathrm{Zr}_{50} \mathrm{Cu}_{35} \mathrm{Al}_{15}$.

The structural evolution on annealing is different for the two samples as well. Although both compositions have a maximum fraction of mixed VP after annealing for 10 minutes, $\mathrm{Zr}_{50} \mathrm{Cu}_{45} \mathrm{Al}_{5}$ becomes more icosahedral and less crystal-like with increasing structural relaxation whereas $\mathrm{Zr}_{50} \mathrm{Cu}_{35} \mathrm{Al}_{15}$ becomes less icosahedral and more crystal-like. In the final state, the crystal-like fraction of $\mathrm{VP}$ in $\mathrm{Zr}_{50} \mathrm{Cu}_{35} \mathrm{Al}_{15}$ is higher than in $\mathrm{Zr}_{50} \mathrm{Cu}_{45} \mathrm{Al}_{5}$, while the icosahedrallike fractions are similar. Taken together, these results suggest that the crystal-like clusters are 

either similar in stability or slightly more stable in $\mathrm{Zr}_{50} \mathrm{Cu}_{45} \mathrm{Al}_{5}$. Higher thermal stability for crystal-like clusters also supports the hypothesis that the crystal-like clusters become more strongly ordered in structure even as they shrink with annealing.

One possible origin for crystal-like order is the fluctuations of embryos leading up to nucleation in the cooling liquid. In that case, stronger crystal-like order may indicate that the cooling liquid came closer to nucleation and crystallization. Similarly, crystal-like order that is more stable against thermal annealing in the glassy state may indicate lower relative free energy of the crystal-like structure with respect to the icosahedral-like structure, although it could also result from lower atomic mobility.

These results suggest an important role for destabilizing crystal-like structures and enhacing glass formation. Most structurally-based concepts for increasing GFA focus on increasing the stability of the glass structure by efficient short-range packing $[9,10,12]$ or by increasing icosahedral order inconsistent with translational symmetry $[13,52]$. Our results suggest that the primary difference between $\mathrm{Zr}_{50} \mathrm{Cu}_{45} \mathrm{Al}_{5}$, a good glas former, and $\mathrm{Zr}_{50} \mathrm{Cu}_{35} \mathrm{Al}_{15}$, a less good glass former, is better stability of crystal-like structures in the poor glass former. A lower free energy crystal-like state will favor nucleation and growth of the crystal phase, preventing glass formation. GFA criteria based on thermal characteristics like $\Delta T_{x}$ or $\gamma$ or on phase diagrams like searching for deep eutectics or low liquidus regions (e.g. [53]) implicitly include low stability of the competing crystalline phases. Our results emphasize the structural results of limiting crystal stability and demonstrate an approach to characterizing this contribution to glass stability in amorphous samples, without complete crystallization.

\section{Conclusions}

A combination of FEM experimental data, HRMC structure refinements, and analysis of the resulting models shows that in the $\mathrm{Zr}-\mathrm{Cu}-\mathrm{Al}$ alloy system, a poorer glass former, $\mathrm{Zr}_{50} \mathrm{Cu}_{35} \mathrm{Al}_{15}$ has more pronounced and more thermally stable crystal-like medium-range order than a better glass former, $\mathrm{Zr}_{50} \mathrm{Cu}_{45} \mathrm{Al}_{5}$. Peaks in the experimental FEM data are connected to structure type, crystal-like or icosahedral, based on examining the structure of sub-models of HRMC-derived structures created by locating highly diffracting, planar structures via the threedimensional Fourier transform of the model. With increasing structural relaxation due to thermal annealing below $T_{g}$, the $\mathrm{Zr}_{50} \mathrm{Cu}_{35} \mathrm{Al}_{15}$ alloy exhibits stronger crystal-like order and weaker icosahedral-like order, the opposite of the behavior for $\mathrm{Zr}_{50} \mathrm{Cu}_{45} \mathrm{Al}_{5}$. However, variableresolution FEM data shows that the length scale associated with crystal-like order decreases with annealing, so the crystal-like regions may be simultaneously shrinking and growing more strongly internally ordered.

The connection between crystal-like medium-range order and glass forming ability suggests that destabilizing crystal-like structure is important to improving the glass forming ability of BMGs in this system. We speculate that the crystal-like order could arise from the fluctuations leading up to nucleation.

\section{Acknowlegments}

This work was supported by the National Science Foundation under Contract No. DMR1205899 and CMMI-1232731 (JJM, PZ, and PMV). The facilities and instrumentation for microscopy and calorimetry were supported by the University of Wisconsin Materials Research Science and Engineering Center (DMR-1121288). The synthesis of alloys was supported by U.S. 
DOE, Office of Basic Energy Sciences, Materials Science and Engineering Division, through the Ames Laboratory (MFB and MJK), Iowa State University under contract DE-AC02-07CH11358. 


\section{References}

[1] C. Hays, C. Kim, W. Johnson, Microstructure controlled shear band pattern formation and enhanced plasticity of bulk metallic glasses containing in situ formed ductile phase dendrite dispersions, Phys. Rev. Lett. 84 (2000) 2901.

[2] R. Vaidyanathan, M. Dao, G. Ravichandran, S. Suresh, Study of mechanical deformation in bulk metallic glass through instrumented indentation, Acta Mater. 49 (2001) 3781-3789.

[3] Z. Bian, G. He, G. L. Chen, Microstructure and mechanical properties of as-cast $\mathrm{Zr}_{52.5} \mathrm{Cu}_{17.9} \mathrm{Ni}_{14.6} \mathrm{Al}_{10} \mathrm{Ti}_{5}$ bulky glass alloy, Scr. Mater. 43 (2000) 1003-1008.

[4] M. Carmo, R. C. Sekol, S. Ding, G. Kumar, J. Schroers, A. D. Taylor, Bulk metallic glass nanowire architecture for electrochemical applications, ACS Nano. 5 (2011) 2979-2983.

[5] A. Inoue, Stabilization of metallic supercooled liquid and bulk amorphous alloys, Acta Mater. 48 (2000) 279-306.

[6] D. Turnbull, Under what conditions can a glass be formed, Contemp. Phys. 10 (1969) 473-488.

[7] Z. P. Lu, C. T. Liu, Glass formation criterion for various glass-forming systems, Phys. Rev. Lett. 91 (2003) 115505.

[8] Z. P. Lu, H. Bei, C. T. Liu, Recent progress in quantifying glass-forming ability of bulk metallic glasses, Intermetallics. 15 (2007) 618-624.

[9] T. Egami, The atomic structure of aluminum based metallic glasses and universal criterion for glass formation, J. Non. Cryst. Solids. 205-207 (1996) 575-582.

[10] D. B. Miracle, A structural model for metallic glasses, Nat. Mater. 3 (2004) 697-702.

[11] D. B. Miracle, The density and packing fraction of binary metallic glasses, Acta Mater. 61 (2013) 3157-3171.

[12] T. Egami, Y. Waseda, Atomic size effect on the formability of metallic glasses, J. Non. Cryst. Solids. 64 (1984) 113-134.

[13] Y. Q. Cheng, E. Ma, H. W. Sheng, Alloying strongly influences the structure, dynamics, and glass forming ability of metallic supercooled liquids, Appl. Phys. Lett. 93 (2008) 111913.

[14] N. Jakse, A. Pasturel, Glass forming ability and short-range order in a binary bulk metallic glass by ab initio molecular dynamics, Appl. Phys. Lett. 93 (2008) 113104. 
[15] H. W. Sheng, W. K. Luo, F. M. Alamgir, J. M. Bai, E. Ma, Atomic packing and short-tomedium-range order in metallic glasses, Nature. 439 (2006) 419-425.

[16] Y. Q. Cheng, H. W. Sheng, E. Ma, Relationship between structure, dynamics, and mechanical properties in metallic glass-forming alloys, Phys. Rev. B. 78 (2008) 014207.

[17] L. Pusztai, E. Sváb, Structure study of $\mathrm{Ni}_{62} \mathrm{Nb}_{38}$ metallic glass using reverse Monte Carlo simulation , J. Non. Cryst. Solids. 156-158 (1993) 973-977.

[18] L. Yang, S. Yin, X. D. Wang, Q. P. Cao, J. Z. Jiang, K. Saksl, H. Franz, Atomic structure in Zr70Ni30 metallic glass, J. Appl. Phys. 102 (2007) 083512.

[19] T. Takagi, T. Ohkubo, Y. Hirotsu, B. S. Murty, K. Hono, D. Shindo, Local structure of amorphous $\mathrm{Zr}_{70} \mathrm{Pd}_{30}$ alloy studied by electron diffraction, Appl. Phys. Lett. 79 (2001) 485.

[20] Y. Q. Cheng, E. Ma, H. W. Sheng, Atomic level structure in multicomponent bulk metallic glass, Phys. Rev. Lett. 102 (2009) 245501.

[21] D. B. Miracle, W. S. Sanders, O. N. Senkov, The influence of efficient atomic packing on the constitution of metallic glasses, Philos. Mag. 83 (2003) 2409-2428.

[22] D. B. Miracle, E. A. Lord, S. Ranganathan, Candidate Atomic Cluster Configurations in Metallic Glass Structures S. Ranganathan, Mater. Trans. 47 (2006) 1737-1742.

[23] D. B. Miracle, O. N. Senkov, A geometric model for atomic configurations in amorphous Al alloys, J. Non. Cryst. Solids. 319 (2003) 174-191.

[24] D. B. Miracle, Efficient local packing in metallic glasses, J. Non. Cryst. Solids. 342 (2004) 89-96.

[25] D. Nelson, Order, frustration, and defects in liquids and glasses, Phys. Rev. B. 28 (1983) 5515.

[26] A. Hirata, L. J. Kang, T. Fujita, B. Klumov, K. Matsue, M. Kotani, A. R. Yavari, M. W. Chen, Geometric frustration of icosahedron in metallic glasses, Science. 341 (2013) 376379 .

[27] J. P. K. Doye, D. J. Wales, The Structure and Stability of Atomic Liquids: From Clusters to Bulk, Science. 271 (1996) 484-487.

[28] J. P. K. Doye, D. J. Wales, F. H. M. Zetterling, M. Dzugutov, The favored cluster structures of model glass formers, J. Chem. Phys. 118 (2003) 2792.

[29] F. H. M. Zetterling, M. Dzugutov, S. I. Simdyankin, Formation of large-scale icosahedral clusters in a simple liquid approaching the glass transition, J. Non. Cryst. Solids. 293-295 (2001) 39-44. 
[30] M. Dzugutov, S. Simdyankin, F. Zetterling, Decoupling of Diffusion from Structural Relaxation and Spatial Heterogeneity in a Supercooled Simple Liquid, Phys. Rev. Lett. 89 (2002) 195701.

[31] Z. P. Lu, C. T. Liu, Role of minor alloying additions in formation of bulk metallic glasses: A review, J. Mater. Sci. 39 (2004) 3965-3974.

[32] D. B. Miracle, The efficient cluster packing model - An atomic structural model for metallic glasses, Acta Mater. 54 (2006) 4317-4336.

[33] J. Hwang, Z. H. Melgarejo, Y. E. Kalay, I. Kalay, M. J. Kramer, D. S. Stone, P. M. Voyles, Nanoscale Structure and Structural Relaxation in $\mathrm{Zr}_{50} \mathrm{Cu}_{45} \mathrm{Al}_{5}$ Bulk Metallic Glass, Phys. Rev. Lett. 108 (2012) 195505.

[34] D. Wang, H. Tan, Y. Li, Multiple maxima of GFA in three adjacent eutectics in $\mathrm{Zr}-\mathrm{Cu}-\mathrm{Al}$ alloy system - A metallographic way to pinpoint the best glass forming alloys, Acta Mater. 53 (2005) 2969-2979.

[35] D. Okai, M. Inoue, T. Mori, T. Fukami, T. Yamasaki, H. M. Kimura, A. Inoue, Annealing effect on mechanical constants for $\mathrm{Ca}_{48} \mathrm{Mg}_{27} \mathrm{Cu}_{25}$ bulk metallic glass, J. Alloys Compd. 504 (2010) S95-S97.

[36] J. Hwang, Nanometer Scale Atomic Structure of Zirconium Based Bulk Metallic Glass, Dissertation. University of Wisconsin-Madison (2011).

[37] W. G. Stratton, J. Hamann, J. H. Perepezko, P. M. Voyles, X. Mao, S. V. Khare, Aluminum nanoscale order in amorphous $\mathrm{Al}_{92} \mathrm{Sm}_{8}$ measured by fluctuation electron microscopy, Appl. Phys. Lett. 86 (2005) 141910.

[38] P. M. Voyles, J. M. Gibson, M. M. J. Treacy, Fluctuation microscopy: a probe of atomic correlations in disordered materials, J. Electron Microsc. 49 (2000) 259-266.

[39] J. Hwang, P. Voyles, Variable resolution fluctuation electron microscopy on $\mathrm{Cu}-\mathrm{Zr}$ metallic glass using a wide range of coherent STEM probe size, Microsc. Microanal. 17 (2011) 67-74.

[40] P. M. Voyles, D. A. Muller, Fluctuation microscopy in the STEM, Ultramicroscopy. 93 (2002) 147-159.

[41] D. T. Schweiss, J. Hwang, P. M. Voyles, Inelastic and elastic mean free paths from FIB samples of metallic glasses, Ultramicroscopy. 124 (2013) 6-12.

[42] J. M. Gibson, M. M. J. Treacy, P. M. Voyles, Atom pair persistence in disordered materials from fuctuation microscopy, 83 (2000) 169-178. 
[43] T. C. Petersen, I. Yarovsky, I. Snook, D. G. Mcculloch, G. Opletal, Structural analysis of carbonaceous solids using an adapted reverse Monte Carlo algorithm, Carbon. 41 (2003) 2403-2411.

[44] G. Opletal, T. C. Petersen, B. O’Malley, I. Snook, D. Mcculloch, I. Yarovsky, HRMC: Hybrid Reverse Monte Carlo method with silicon and carbon potentials, Comput. Phys. Commun. 178 (2008) 777-787.

[45] G. Opletal, T. C. Petersen, B. O'Malley, I. K. Snook, D. G. McCulloch, I. Yarovsky, HRMC_1.1: Hybrid Reverse Monte Carlo method with silicon and carbon potentials, Comput. Phys. Commun. 182 (2011) 542.

[46] M. M. J. Treacy, K. B. Borisenko, The Local Structure of Amorphous Silicon, Science. 335 (2012) 950-953.

[47] R. K. Dash, P. M. Voyles, J. M. Gibson, M. M. J. Treacy, P. Keblinski, A quantitative measure of medium-range order in amorphous materials from transmission electron micrographs, J. Phys. Condens. Matter. 15 (2003) S2425-S2435.

[48] H. Sheng, https://sites.google.com/site/eampotentials/Home/ZrCuAl, 10-15-2014.

[49] S. Le Roux, P. Jund, Ring statistics analysis of topological networks: New approach and application to amorphous $\mathrm{GeS}_{2}$ and $\mathrm{SiO}_{2}$ systems, Comput. Mater. Sci. 49 (2010) 70-83.

[50] S. G. Hao, M. J. Kramer, C. Z. Wang, K. M. Ho, S. Nandi, A. Kreyssig, A. I. Goldman, V. Wessels, K. K. Sahu, K. F. Kelton, R. W. Hyers, S. M. Canepari, J. R. Rogers, Experimental and ab initio structural studies of liquid $\mathrm{Zr}_{2} \mathrm{Ni}$, Phys. Rev. B. 79 (2009) 104206.

[51] Y. Q. Cheng, A. J. Cao, H. W. Sheng, E. Ma, Local order influences initiation of plastic flow in metallic glass: Effects of alloy composition and sample cooling history, Acta Mater. 56 (2008) 5263-5275.

[52] J. Ding, Y. Q. Cheng, E. Ma, Full icosahedra dominate local order in $\mathrm{Cu}_{64} \mathrm{Zr}_{34}$ metallic glass and supercooled liquid, Acta Mater. 69 (2014) 343-354.

[53] H. Cao, D. Ma, K. C. Hsieh, L. Ding, W. G. Stratton, P. M. Voyles, Y. Pan, M. Cai, J. T. Dickinson, Y. A. Chang, Computational thermodynamics to identify $\mathrm{Zr}-\mathrm{Ti}-\mathrm{Ni}-\mathrm{Cu}-\mathrm{Al}$ alloys with high glass-forming ability, Acta Mater. 54 (2006) 2975-2982. 

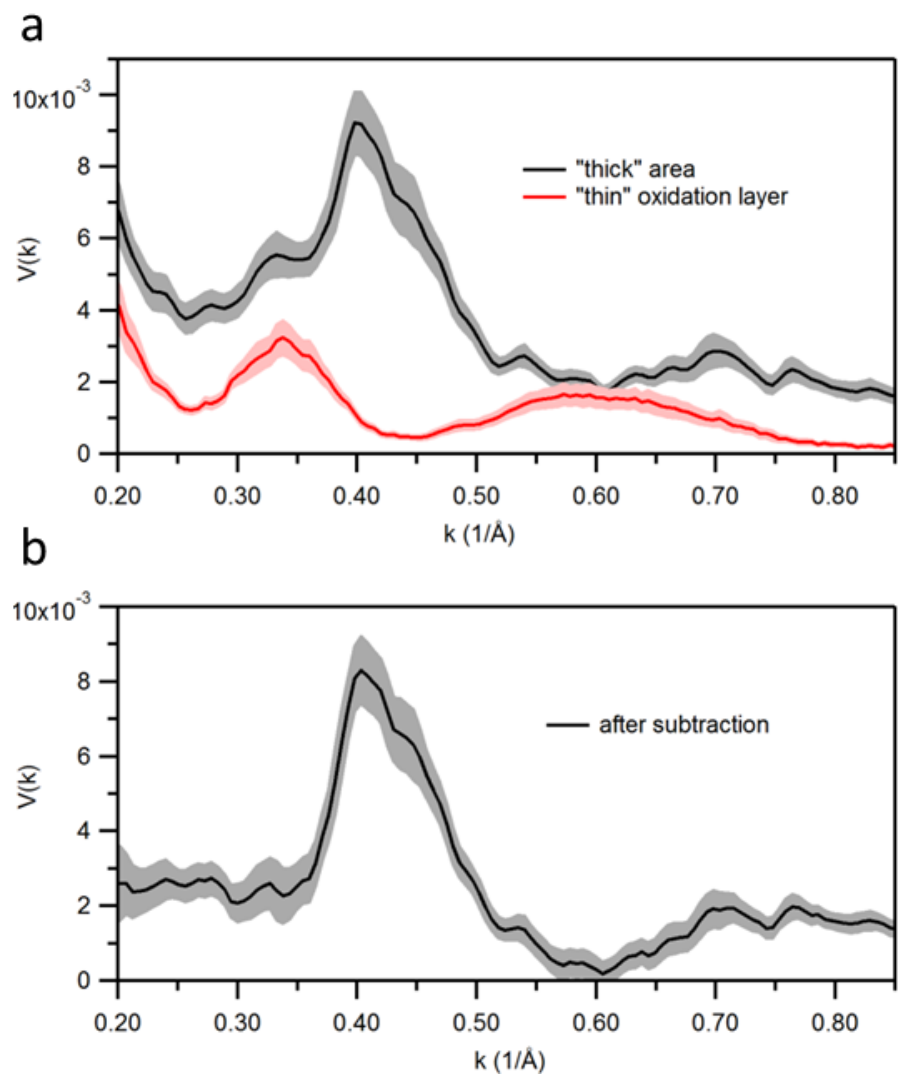

Figure 1. a) $V(k)$ from thick area and thin oxidation layer of as-cast $\mathrm{Zr}_{50} \mathrm{Cu}_{35} \mathrm{Al}_{15}$ BMG. b) $V(k)$ after subtracting off the contribution of the oxidation layer. 

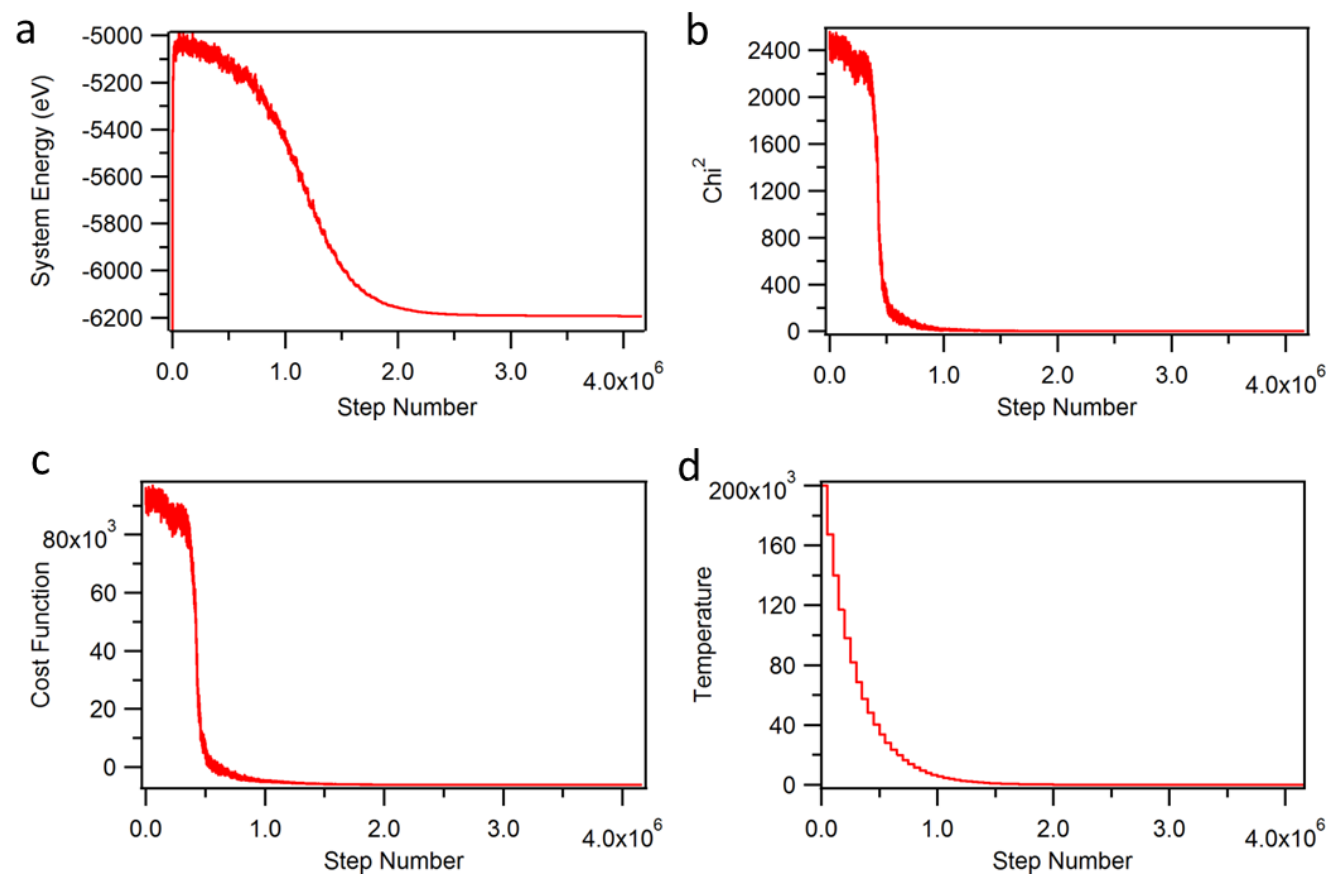

Figure 2. a) System energy, b) $\chi^{2}$, c) cost function, and d) temperature as a function of step number for the $300{ }^{\circ} \mathrm{C}, 60 \mathrm{~min}$ annealed HRMC simulation. 


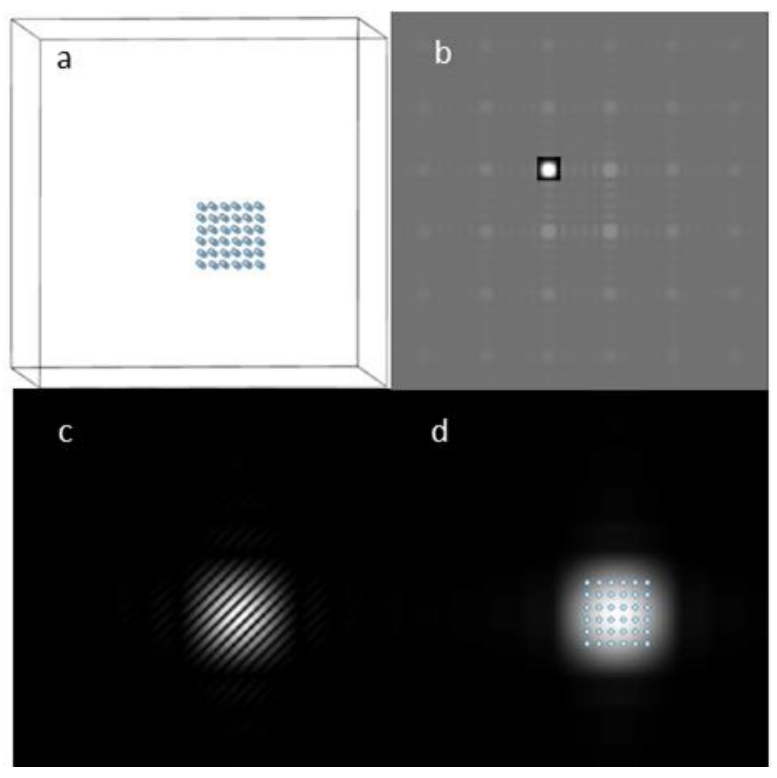

Figure 3. All images are 2D projections of 3D images. a) An aluminum FCC nanocrystal inside a larger box; b) FT of model in (a) with a (111) spot selected for filtering (the symmetric reflection, also selected, is in a different plane); c) IFT of the selected spot in (b) (and its symmetric reflection); d) image (c) after smoothing overlayed with the original model position. 


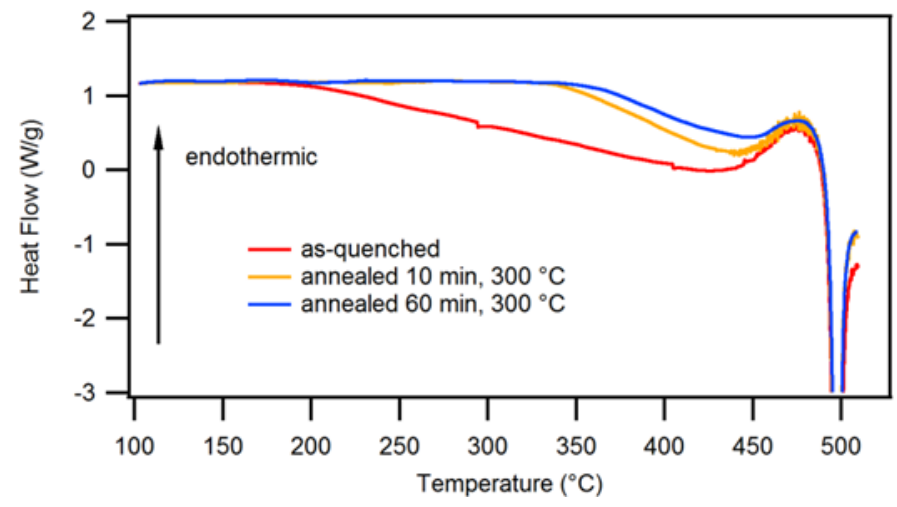

Figure 4. DSC data for as quenched and annealed samples. 

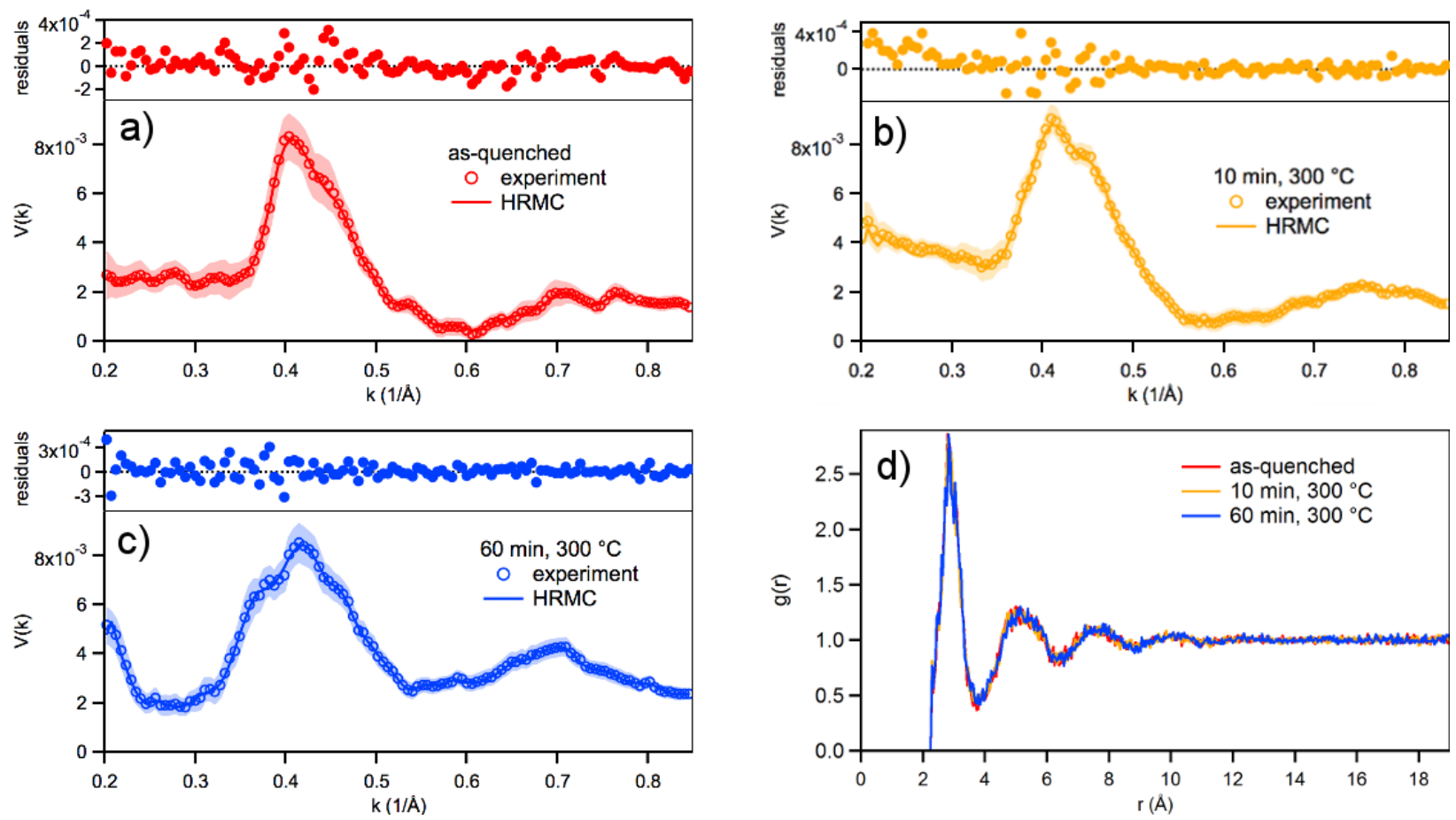

Figure 5. a-c) Simulated $V_{s}(k)$ for all three sample states with residuals plotted above show excellent agreement with experimental $V_{e}(k)$. d) $g(r)$ for all sample states. 
a

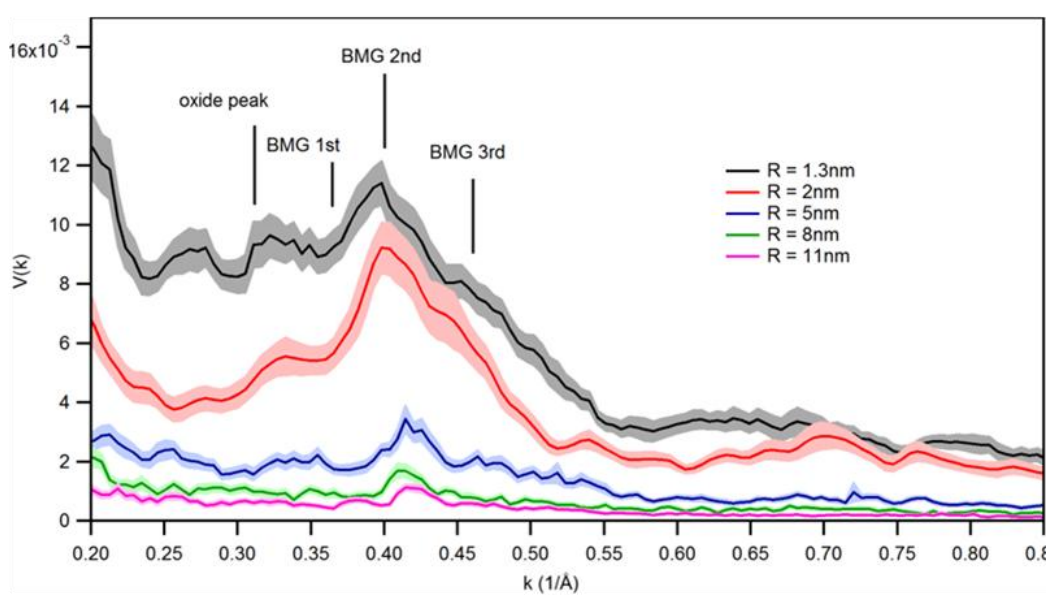

b
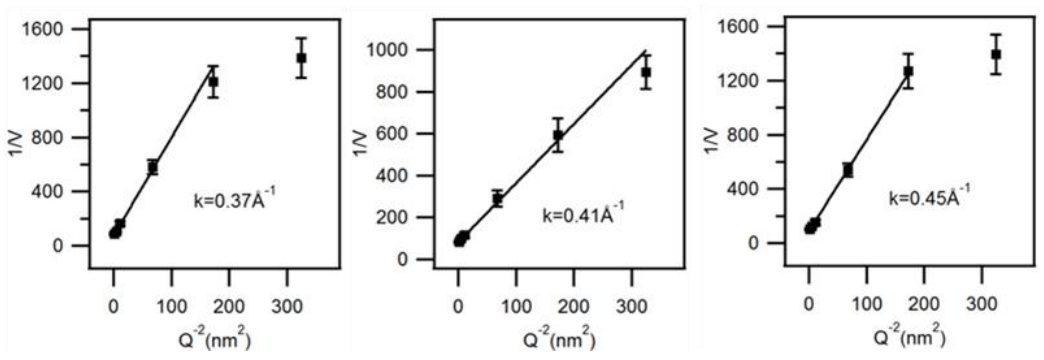

Figure 6. a) VRFEM plots at five resolutions. The peak at $k \sim 0.37 \AA^{-1}$ arises from the oxidation layer and the other three peaks due to the BMG. b) The fitting of the decay length $\Lambda$ for three BMG peaks in (a) using the pair persistent model. 
a

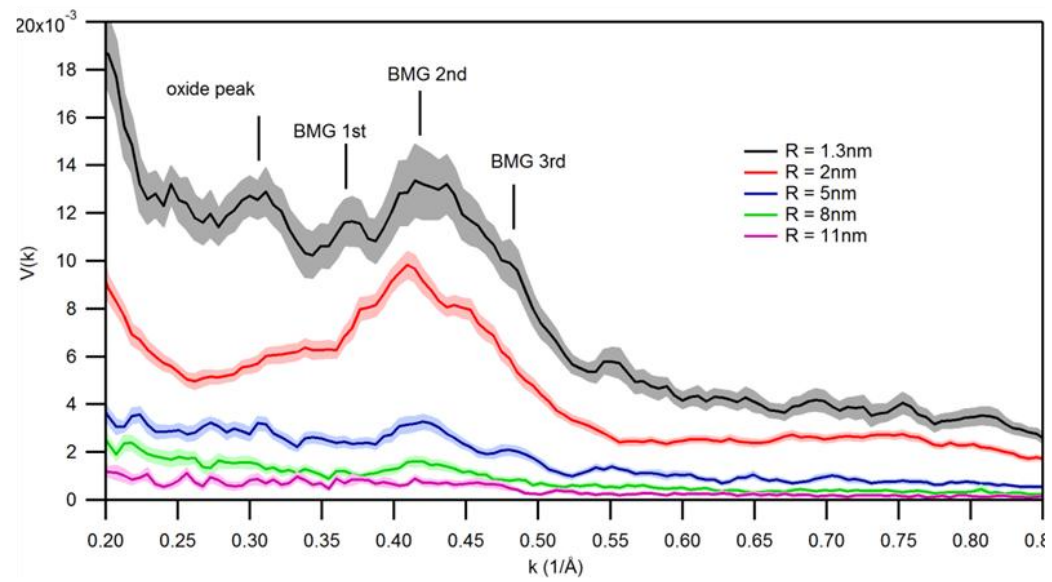

b
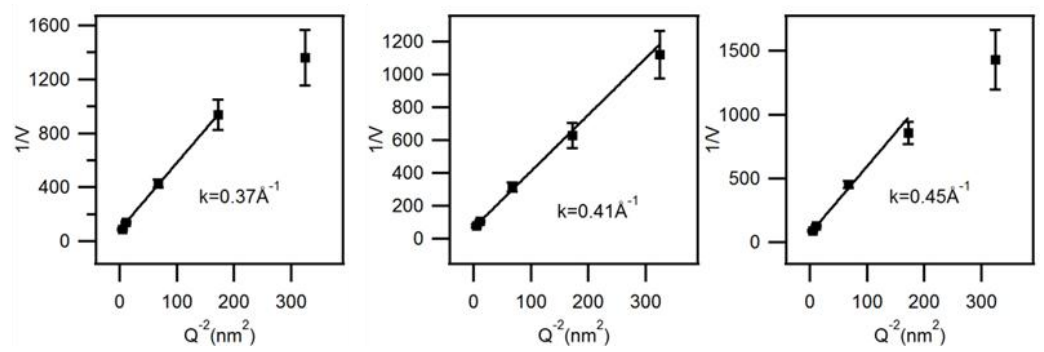

Figure 7. VRFEM plots at five resolutions for the sample annealed at $0.83 T_{g}$ for $10 \mathrm{~min}$. 
a

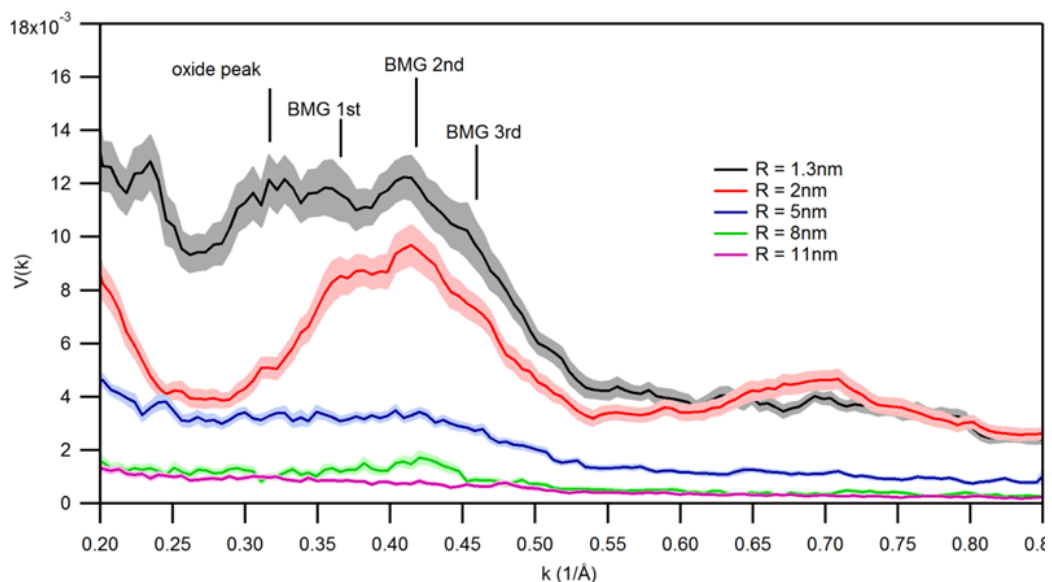

b
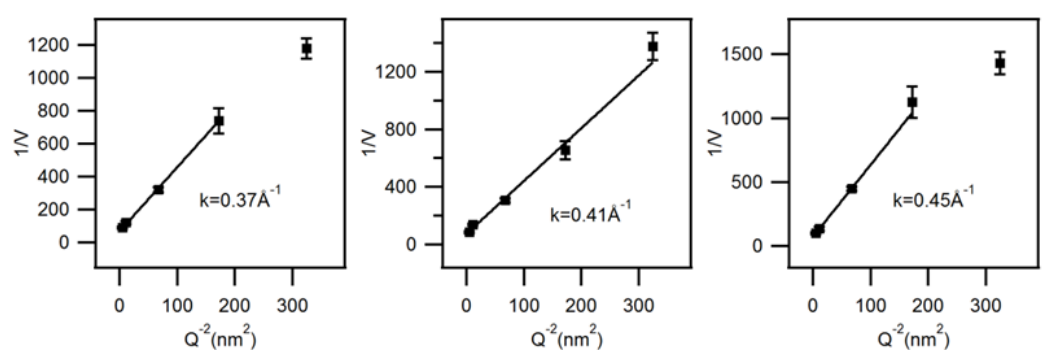

Figure 8. VRFEM plots at five resolutions for the sample annealed at $0.83 T_{g}$ for $60 \mathrm{~min}$. 


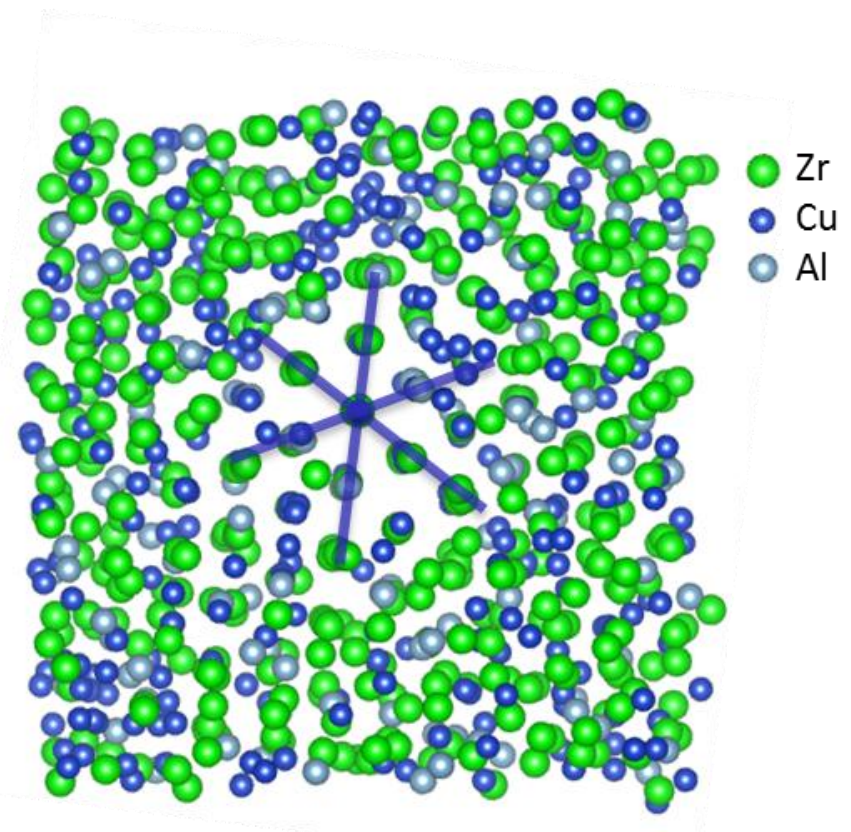

Figure 9. A region of the $60 \mathrm{~min}, 300{ }^{\circ} \mathrm{C}$ final model illustrating the contrasting planar and glassy features of the models. 


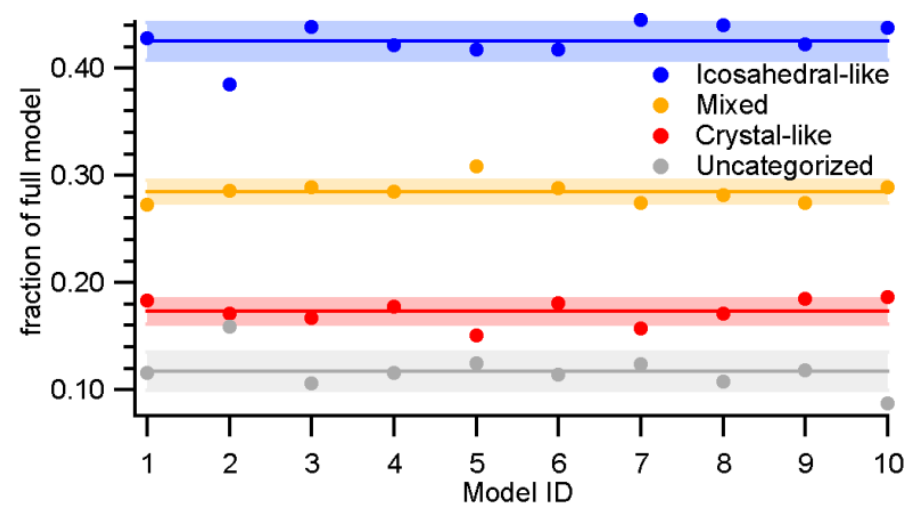

Figure 10. VP fractions for ten HRMC simulations against the most annealed data, starting from different random number generator seeds yield statistically similar results. The solid lines are the mean value of all ten simulations, and the colored bands are one standard deviation of the mean. 

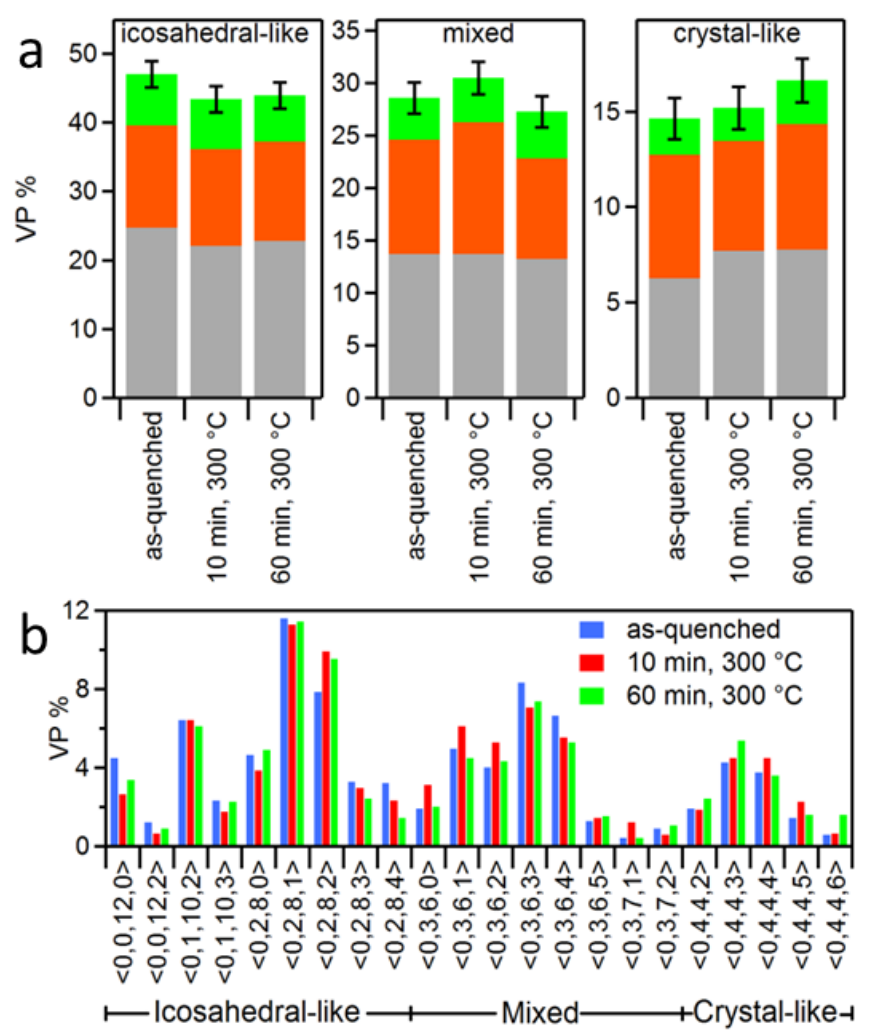

Figure 11. (a) Histograms of the VP categories for the HRMC models. From top to bottom within each bar: Al-centered (green), Cu-centered (orange), Zr-centered (gray) VPs. (b) Histogram of VP indices for the most common indices in the model. Bottom axis is sorted by VP type. 

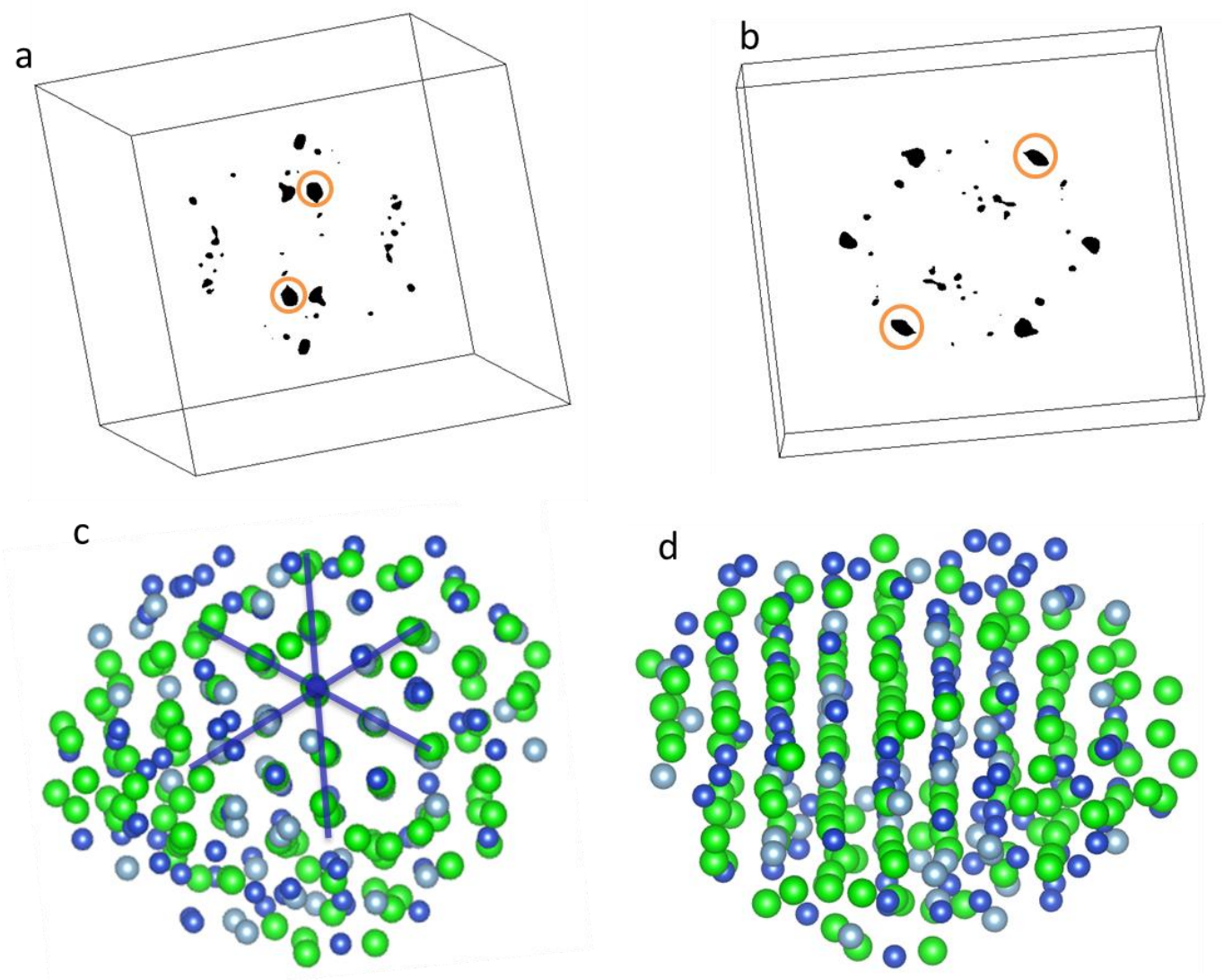

Figure 12. a-b) Two orientations of an isosurface plot of the spots in reciprocal space for the 60 min, $300{ }^{\circ} \mathrm{C}$ annealed sample. c) The sub-model that results from the spot-pair circled in (a) and (b). d) The sub-model in (c) reoriented corresponding to the $\boldsymbol{g}$ direction to show the planes of atoms (vertical) that give rise to Bragg diffraction. 


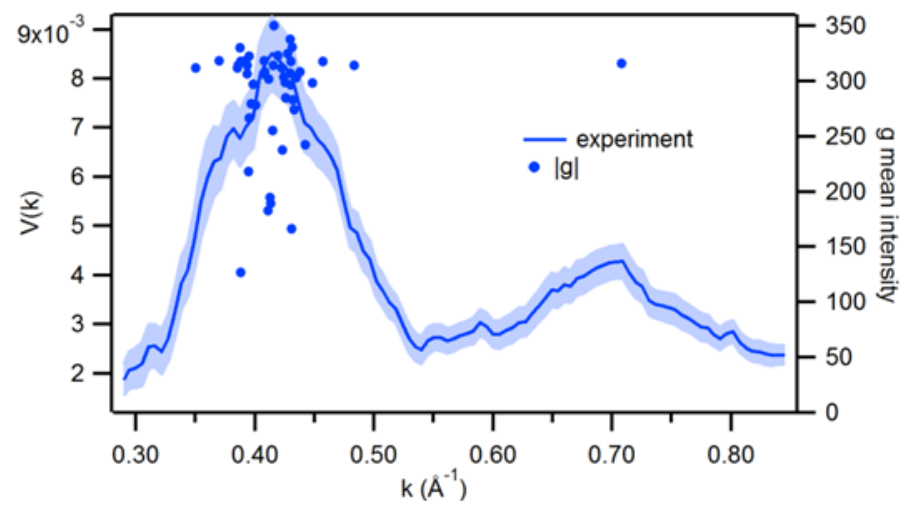

Figure 13. The length of each $\mathbf{g}$ identified in the computed reciprocal space with respect to $V_{e}(k)$ for the as-quenched sample. 


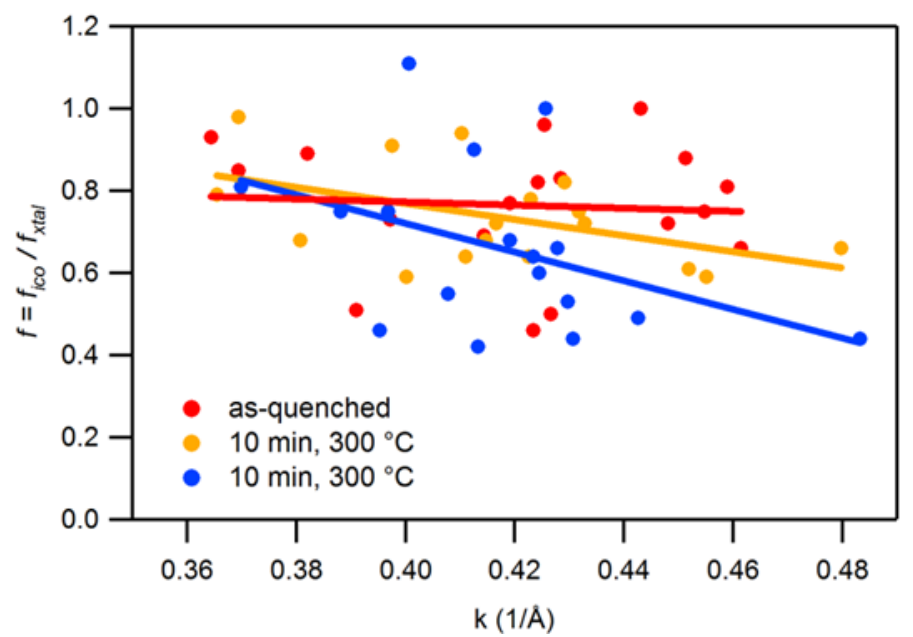

Figure 14. Fits to $f=f_{\text {ico }} / f_{\text {xtal }}$. Solid lines are fits to the data points. 


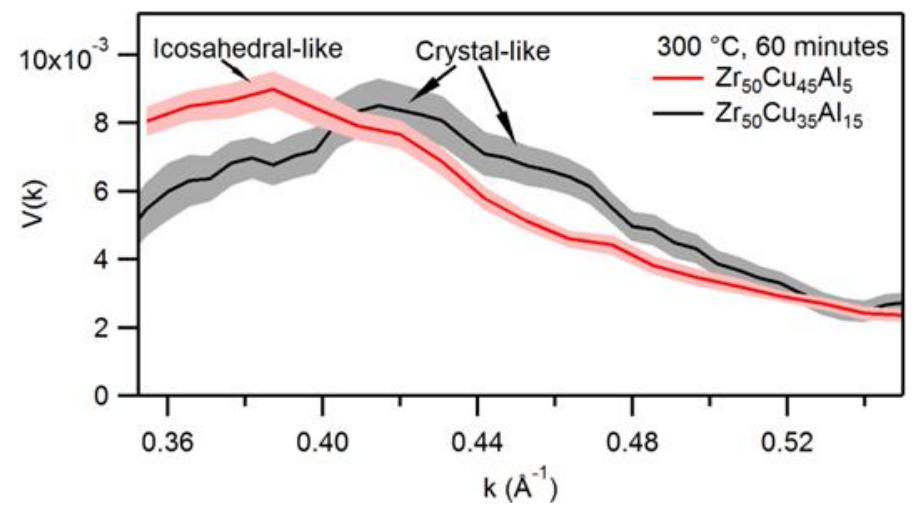

Figure 15. $\mathrm{Zr}_{50} \mathrm{Cu}_{35} \mathrm{Al}_{15}$ and $\mathrm{Zr}_{50} \mathrm{Cu}_{45} \mathrm{Al}_{5} V(k)$ for the $300{ }^{\circ} \mathrm{C}, 60$ min annealed sample. 
Table 1

The corresponding parameters for five different FEM resolutions.

\begin{tabular}{llll}
\hline FEM Resolution $(\mathrm{nm})$ & C2 Aperture (um) & Conv. Angle (mrad) & Spot number \\
\hline 1.3 & 10 & 1.17 & 7 \\
2.0 & 10 & 0.75 & 7 \\
5.0 & 10 & 0.30 & 6 \\
8.0 & 10 & 0.20 & 5 \\
11.0 & 5 & 0.14 & 4 \\
\hline
\end{tabular}


Table 2

Voronoi polyhedra indices of icosahedral-like, crystal-like, and mixed categories.

\begin{tabular}{lll}
\hline Icosahedral-like & Crystal-like & Mixed \\
\hline$\langle 0,0,12, *\rangle$ & $\langle 0,4,4, *\rangle$ & $<0,3,6, *\rangle$ \\
$\langle 0,2,8, *\rangle$ & $\langle 0,5,2, *\rangle$ & $<0,3,7,2\rangle$ \\
$\langle 0,1,10, *\rangle$ & $\langle 0,6,0,8\rangle$ & $<1,2,5,4\rangle$ \\
$\langle 1,0,9,3\rangle$ & $<0,6,0,2\rangle$ &
\end{tabular}


Table 3

Decay lengths for as-cast and annealed samples.

\begin{tabular}{llll}
\hline Decay length & As-cast & Annealed $300{ }^{\circ} \mathrm{C}, 10 \mathrm{~min}$ & Annealed $300{ }^{\circ} \mathrm{C} 60, \mathrm{~min}$ \\
\hline$k=0.45 \AA^{-1}$ & $0.59 \pm 0.06 \mathrm{~nm}$ & $0.56 \pm 0.06 \mathrm{~nm}$ & $0.56 \pm 0.06 \mathrm{~nm}$ \\
$k=0.41 \AA^{-1}$ & $0.86 \pm 0.06 \mathrm{~nm}$ & $0.69 \pm 0.05 \mathrm{~nm}$ & $0.71 \pm 0.05 \mathrm{~nm}$ \\
$k=0.37 \AA^{-1}$ & $0.51 \pm 0.06 \mathrm{~nm}$ & $0.67 \pm 0.05 \mathrm{~nm}$ & $0.66 \pm 0.02 \mathrm{~nm}$ \\
\hline
\end{tabular}


Table 4

Final energies for all three simulated sample states compared to MD simulation.

\begin{tabular}{llll}
\hline Model & eV/atom & eV/atom above MD & kT at RT above MD \\
\hline MD & -5.06054 & & \\
Starting model & -5.006 & 0.055 & 2.13 \\
t1 & -4.968 & 0.093 & 3.61 \\
t2 & -4.954 & 0.11 & 4.16 \\
t3 & -4.955 & 0.11 & 4.12 \\
\hline
\end{tabular}



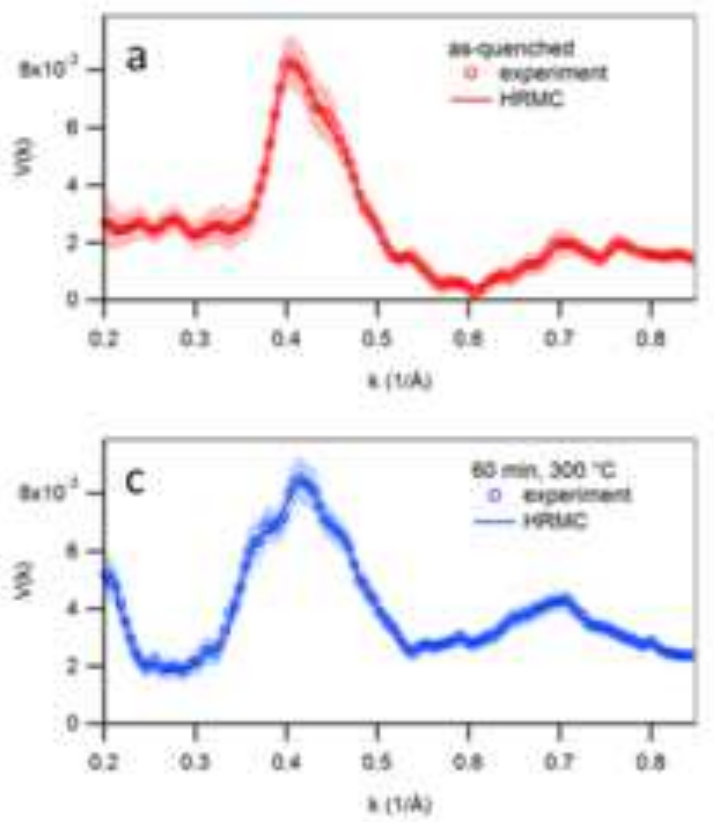

Variance vs $k$ from FEM experiments: (a) as quenched, (b) annealed at $300^{\circ} \mathrm{C}$ for $10 \mathrm{mins}$, (c) annealed at $300^{\circ} \mathrm{C}$ for $60 \mathrm{mins}$. Simulated $V_{3}(k)$ for all three sample states shows excellent agreement with experimental $V_{f}(k)$, d) $g(r)$ for all sample states.

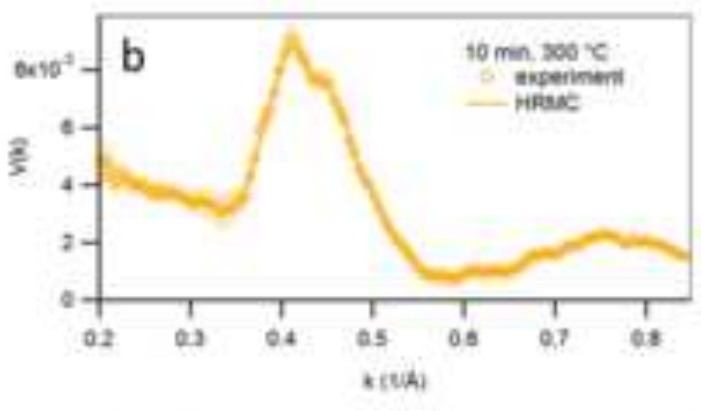

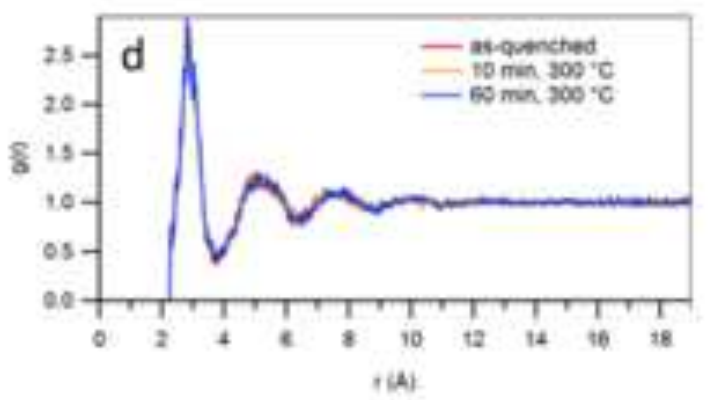

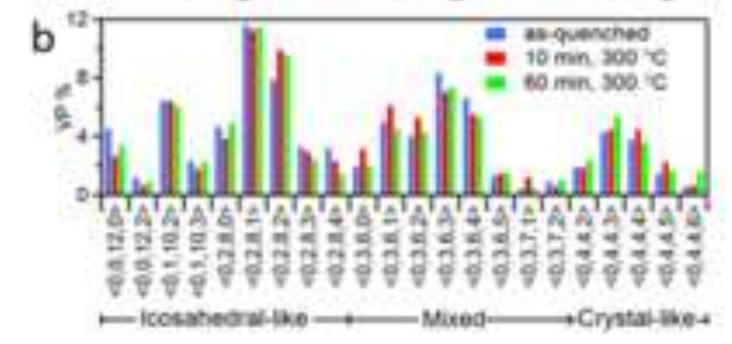

HRMC models: (a) categories for Al-centered (green), Cu-centered (orange), Zr-centered (gray)

VPs. (b) VP indices for the most common indices.

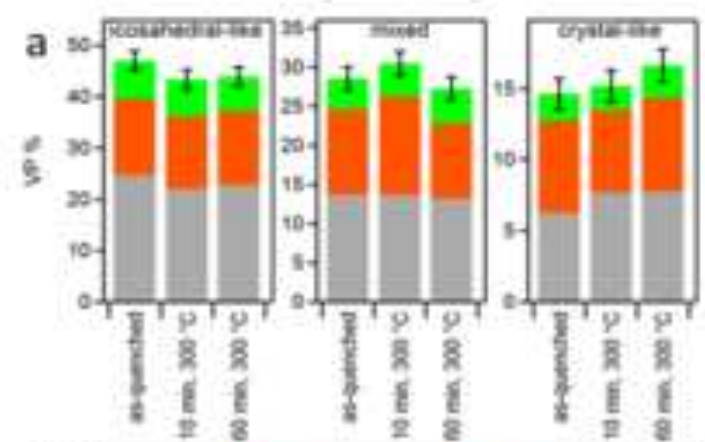

